\title{
Highlights of ASCO
}

\author{
Frank Griesinger* \\ Department of Hematology and Oncology, University Internal Medicine-Oncology, Germany
}

Submission: September 10, 2019; Published: October 10, 2019

*Corresponding author: Frank Griesinger, Department of Hematology and Oncology, University Internal Medicine-Oncology, Germany

\section{Introduction}

The Immune-Checkpoint Inhibitor (ICI) therapy that has evolved as a standard of care for stage IV and III is now moving into the early stages. Three studies on ICI-induction therapy in early or locally advanced NSCLC (stages II and III) were presented at ASCO. Induction therapy in stage II and III is deemed to be as effective as adjuvant therapy in these stages, based on meta-analyses. Advantages of induction therapy is the possibility o therapy monitoring, the higher therapy compliance and feasibility during induction $(90 \%$ of 3 full dose cycles in the NATCH trial during induction vs. $60 \%$ of full dose cycles in the adjuvant setting) and early treatment of potential micro metastases. Additionally, in the context of ICI, induction concepts open a window of opportunity for correlative and translational studies on the ample tumor material obtained at resection. Induction therapy is deemed standard of care especially in those patients which are prone for postoperative complications (comorbidities, pneumonectomy) potentially precluding adjuvant therapy.

The first ICI-study was performed with atorolimumab single agent and presented by Kwiatkowski [1]. The presented data were an interim analysis of 101 pts with stages IB toss IIIB, which were included irrespective of PD-L1 expression status. Primary endpoint of the study was "major pathologic remission" as defined by less than $10 \%$ vital tumor cells after 2 administrations of atorolimumab. The MPR rate was 15 /82 (18\%), 4 pts achieved a PCR (Pathologic Complete Remission with no residual vital tumor cells). The MPR rate of PD-L1 positive patients was higher than the one of PD-L1 negative patients $(2 / 26$ vs. $10 / 35(\mathrm{p}=0,055))$, additionally the MPR rate was numerically higher in PD-L1>50\% vs. PD-L1 <50\% $(7 / 29$ vs. $5 / 44(p=0,040))$. Accrual of the study is ongoing with an aim of 180pts. The second induction therapy study was the NEOSTAR trial, which included patients with stages I- IIIA (some N2 patients) irrespective of PD-L1 status to be treated with either single agent Nivolumab or Nivolumab and Ipilimumab. Primary endpoint was MPR-rate, stage IIIA was seen in $20 \%$ of patients. Of 44 included pts. 34 went to surgery, in this cohort, the MPR-rate in the Nivolumab-arm was 2/23 (20\%) and in the
Nivo-Ipi-arm 6/16 (43\%). The MPR-rate correlated with PD-L1 expression [2].

The 3rd induction therapy study (NADIM trial), which was presented as a poster, had, at least in the opinion of the author, the highest impact on the future management of patients [3]. Pts with stage IIIA were included, all patients received 3 cycles of an induction chemotherapy and ICI therapy (Paclitaxel, Carboplatin, Nivolumab). Of 46 recruited patients, 41 went to surgery, the MPR rate relative to the 41 operated or the ITT population ( $\mathrm{n}=46)$ was 35 (85 vs $76 \%)$ respectively). The PCR rate was 25 ( 61 vs. 54\%). These pathologic remission rates have not been seen with any induction modality and if confirmed in larger studies, would represent a breakthrough in the treatment of locally advanced NSCLC. As of yet, it is unclear whether MPR and PCR rates generated with chemotherapy and ICI will be as predictive for recurrence free survival and overall survival as this has been demonstrated for chemotherapy alone or for chemoradiotherapy. For sure, these data will increase the interest in the currently recruiting induction therapy trials with Nivolumab, Pembrolizumab and Atorolimumab that compare chemo-ICI vs. chemotherapy alone in the induction therapy setting. In the mind of the author, the most promising combination would be chemotherapy in combination with ICI, whether ICI single agent therapy will turn out as an effective option in the curative setting will have to be shown.

Durvalumab in the consolidation setting after a radiochemotherapy in non-operable stages II is an established therapy and has gained EMA approval for the subgroup of PDL1 $>1 \%$ positive patients based on a post-hoc EMA required subgroup analysis in the European countries with the exception of Switzerland. Three-year data of the Pacific trial were presented at ASCO, which confirmed the positive impact of durvalumab consolidation for PFS and OS The OS was statistically significantly improved for the experimental arm with a HR of 0.69 , the OS for the PD-L1>1\% population was improved with a HR of 0.59. Therefore, it is highly recommended to test patients in stage III that receive a radio-chemotherapy for the PD-L1 
expression and to treat patients that have responded on radiochemotherapy, that do not have any pneumonitis and that are PD-L1>1\% positive with durvalumab consolidation therapy. The start of the durvalumab therapy should not be delayed for much longer than 2 weeks after the end of radio-chemotherapy, as the data in this group is most abundant and this cohort of patients seems to derive the most important benefit from the therapy [4]. The combination of concurrent ICI, chemotherapy and radiotherapy is currently being explored in clinical trials. In the metastatic situation, the combined ICI-chemotherapy has been established as standard of care based on the Keynote (KN) 189 (non-squamous (non-squ) NSCLC) and the KN 407 (squamous NSCLC). Patients with a PD-L1 expression of $>50 \%$ may also be treated with pembrolizumab single agent therapy based on the KN 24 and 42. Since 2019, the "quadruple combination therapy" with Paclitaxel, Carboplatin, Bevacizumab and Atorolimumab has been approved by the EMA for the therapy of non-squ NSCLC, however the OS data are not as convincing as the OS data generated in the KN 189 trial. However, in the prespecified subgroup of patients with liver metastases, a significant benefit for OS was demonstrated with Pacli-Carbo-Bev-Atezo vs. the Sandler control arm with a HR of 0.52 , suggesting a singular signal in this specific patient population. It is unfortunately still unclear, how important the metastatic burden in the liver was, it is only known that patients with liver metastases had a median of 3 metastases (all organs included) and had a median diameter of metastases of $10 \mathrm{~cm}$. In conclusion this analysis confirms the specific effect of the quadruple combination on liver metastases, therefore these data should be discussed with patients that do have liver metastases and no contraindication against bevacizumab [5].

The Keynote 189 trial was updating das well at ASCO 2019: the coprimary endpoint PFS had not been positive in the primary presentation of data for the subgroup of patients with a PD-L1 expression of $<1 \%$, although an OS benefit had already been seen in this cohort. In the updated data analysis, all coprimary endpoints were now statistically significant, including the PFS in the PD-L1 <1\% cohort with a HR of 0.64 and a $95 \%$ confidence interval of $0.47-0.89$. For the first time, the surrogate parameter PFS2 was presented and was shown to be statistically significantly improved in the ICI-chemotherapy arm. The OS remained statistically significantly and clinically relevantly improved in all subgroups for the combination compared with the chemotherapy control: PD-L1<1\%, PD-L1 1-49\%, PDL1 $>50 \%$ : HR of 0.64 (0.47-0.89), $0.51(0.36-0.73)$ and 0.36 (0.26-0.51 respectively) [6]. The biomarker PD-L1 is used for the stratification of patients to approve therapies, however it has short-comes in terms of prediction of ORR, PFS an OS. Therefore, it is of high interest to evaluate further potential positive, but also negative predictive markers to determine the efficacy of ICI or ICI + chemotherapy. STK11/LKB1 mutations have been shown to have a negative predictive value for the efficacy of ICI single agent therapy especially in the KRAS $\mathrm{mt}+$ population. No data as of yet had been published for STK11/LKB1 mutations as a predictive marker for ICI-chemotherapy combination. In a retrospective analysis, 377 patients having been treated with ICIchemotherapy, were analysed for STK11/LKB1 mutations. 102 patients had an STK11/LKB1 mutation. The ORR, PFS and OS in the $\mathrm{mt}+$ group was significantly inferior compared to the STK11/ LKB1 WT group: PFS 4,8 vs. 7.2 months, OS 10.6 vs. 16.7 months, ORR 32.6 vs. $44.7 \%$. All differences were highly significantly. The STK11/LKB1mt+ group was further compared with a STK11/LKB1mt+ historic control group that had been treated solely with chemotherapy. In this indirect comparison, MOS and MPFS were not improved by the addition of pembrolizumab to chemotherapy: MPFS $4.8 \mathrm{~m}$ vs. $4.3 \mathrm{~m}$, HR 1,13, 95\% CI 0.83 to $1,54, p=0,75$, MOS 10.6 m vs. 10.3 months, HR 1.03, 95\% CI 0.71 to $1.49, \mathrm{p}=0.79$ ) [7].

The highlights of the molecularly defined NSCLC involved three driver mutation (RET, MET, KRAS) and EGFR-TKI therapy in combination with antiangiogenic therapy or with chemotherapy. KRAS mutations is one of the most frequent driver mutations in NSCLC with an incidence of 30 to $40 \%$ of all NSCLC, mainly non-squ NSCLC, highly correlated with exhalative smoking. AMG510 is a new mutation specific, G12C, KRAS inhibitor, first data of a phase I/II study were presented at ASCO for non-squ NSCLC and colorectal cancer: of all KRAS mt+ NSCLC, about 30\% carry a G12C, therefore the frequency of this mutation is about as high as EGFR mutations. 10 patients with NSCLC and KRAS G12C were included in the trial, all had received a minimum of 2 prior therapies. The ORR was $5 / 10$ with $4 / 10$ confirmed responses, the duration of response was 7.,3-27.4 weeks for pts achieving PR, and 8.4 to 25.1 weeks for pts achieving SD. Toxicity was limited to grade 1 and 2 therapy associated AE's. The agent will be available in Germany within clinical trial most likely as of October 2019 [8].

MET Exon 14 Skipping mutations are observed in NSCLC in $3-4 \%$ of cases. Patients tend to be older than the median age of NSCLC and this mutation is not predominantly seen in never smokers. Two agents are currently being studied in Phase II trials and were presented at ASCO. The first agent is Tepotinib, a highly specific MET-inhibitor which is studied in patients that are treatment naive or that have been pretreated with chemo and or immune-checkpoint-inhibitor therapy, but patients must not have been treated with a MET-inhibitor. The MET exon 14 skipping mutation can be detected both in tissue and by liquid biopsy. The ORR was consistent in all lines of treatment confirming the strong driver mutation function of MET exon 14 skipping mutations. The PFS (independent review) of all included patients was 9.5 and 10.8 months for tissue and liquid, respectively, the only clinically relevant side effect are edemas, that led to the discontinuation of the drug in 2/87 patients. Tepotinib leads to a high response of brain metastases. The study is being active with the participation of German centers and the study is being opened for patients with a MET-amplification as well [9]. 
The second agent is Capmatinib and data were presented at ASCO. In contrast to the study population in the Tepotinib study, patients were 3 years younger (median age 71 vs. 74years) and the majority of patients were never smokers, suggesting a bias of the tested patient population. The ORR was higher in the therapy naïve patient cohort than in the pretreated (only chemotherapy or immune therapy, as in the Tepotinib trial, no MET inhibitor pretreatment was allowed). A high CNS activity was observed as with tepotinib and as a class effect the most common side effect were edemas. Median PFS in the therapy naive subgroup was 9.7 months and 5.5 months in the pretreated patients [10]. An interesting study addressed the question of primary resistance to MET inhibitors as well as the question of acquired resistance. 16 patients, MET-inhibitor naive and carrying a MET-exon 14 skipping mutation were studied for the presence of MET-RNA. Interestingly, although a MET exon 14 skipping mutation was present, in 5/16 patients no MET-RNA carrying the exon 14 skipping transcript were found and no protein was detectable as well. These patients did not respond to MET-inhibitors. 11/16 patients had the exon 14 skipping mutation on DNA and RNA level as well as protein level and responded to MET inhibitors. This study points to the fact that potentially MET-exon 14 skipping mutation analyses on the DNA-level should be complemented by either testing for the presence of the transcript or the protein. Mechanisms of acquired resistance were equally studied in this patient cohort, demonstrating on- and off-target resistance mechanisms [11].

RET translocations in lung cancer are seldom with an incidence of 1 to $2 \%$ and are mostly located within the never smoker cohort. The treatment options for RET-translocated patients were limited with multi-kinase RET-inhibitors, ORR rates in the range of $25 \%$ and short ( $<6$ months) PFS. In 2018, the data of LOXO 292 were presented at ASCO, which showed a high efficacy in RET-translocated and mutated tumors and a very limited toxicity profile. Thisyear at ASCO, the first data of the agent BLU667 were presented, another specific and highly active RETinhibitor. In a phase II study patients with a RET translocation in NSCLC were included, the ORR rate was between 58 and $60 \%$, in treatment naïve patients it was 71\%, the DCR was > 90\%. 7/9 patients with measurable CNS metastases responded to BLU667, the two no responders did not have a known RET-translocation partner (other than KIF5B or CCDC6). Studies for RET altered patients (RET-translocations as well as RET-mutations) are open for all tumor types including NSCLC, papillary thyroid cancer, colorectal cancer and pancreatic cancer with RET-translocations and medullary thyroid cancer with RET-mutations. Patients with NSCLC should be tested prior to starting 1st line therapy in order to allow inclusion in the study [12].

So called uncommon EGFR mutations are seen in about 10$15 \%$ of EGFR mt+ NSCLC, three distinct groups are distinguished: group I, II (T790M in exon 20) und Gruppe III (insertions in exon 20). Exon 20 insertions are difficult to treat as the configuration of the EGFR receptor ATP binding site is not very different to the wild type configuration, so that EGFR inhibitors demonstrated important EGFR WT toxicities. At ASCO 2019, data for the Exon 20 insertion specific inhibitor TAK788, which shows a higher IC50 for the inhibition of EGFR WT than inhibitor of EGFR exon 20 ins, were presented. The ORR was between 43 und $56 \%$ (Pts with or without primary CNS metastases), the median PFS was 8.1 months for patients without CNS metastases. TAK 788 is currently being rolled out in studies throughout Europe to offer patients, that as of yet could only be treated with chemotherapy, will be treated with a molecular targeted therapeutic agent. Patients therefore should be tested fort the presence of EGFR mutations in Exons 18 to 21 [13].

The standard for 1st line therapy for EGFR "common mutations", i.e. exon 19 deletions and exon 21 mutations are 1st, 2nd or 3rd generation TKI's, since the publication of the Osimertinib data, this 3rd generation TKI is for most doctors the preferred choice based on superior toxicity profile, efficacy (PFS) and high CNS efficacy. Since the ASCO 2018, the question of combining TKI therapy with chemotherapy has been raised with the presentation of the NEJ009 study. Japanese patients with common mutations received either gefitinib followed in second line therapy by a standard of care with Pemetrexed/ Cis or Carboplatin (control arm) or an upfront Gefitinib + Pemetrexed/Cis- or Carboplatin combination. An OS benefit of more than 1 year was demonstrated for the primary combination therapy. Open questions in the trial is whether patients and how many were tested for T790M at acquired resistance and how patients were treated post study. Therefore, this study did not lead to a broad acceptance in the community. At ASCO 2019, another study, which has been published already in the JCO also full paper was presented: this was a monocentric study with a similar concept as in the NEJ009 trial however no fixed recommended post Gefitinib treatment. A doubling of PFS and OS was seen as well as a doubling of toxicity. However, as in the Japanese trial, the post study treatment rate in the Gefitinib arm was only $25 \%$, no data were presented as to testing for T790M or whether $\mathrm{T} 790 \mathrm{M}+$ patients received osimertinib. Therefore, the data generated in this Indian trial cannot be easily transferred to the Caucasian world with completely different health care systems and access to drugs. Therefore, most likely the data of the FLAURA2 study will have to be awaited which will test Osimiertinib vs. Osimertinib + chemotherapy in common EGFR $\mathrm{mt}+$ patients. Perhaps this combination therapy is a very viable option for patients with computations such as p53 mutations where it has been shown that these patients have an inferior ORR, PFS and OS than p53 WT patients on EGFR TKI [14-16].

A second combination approach is TKI + anti-angiogenic treatment. The combination of Erlotinib and Bevacizumab has been approved by the EMA since some years, however because of lack of OS benefit, increase in toxicity and the necessity of regular i.e. treatment in patients that otherwise would be treated only with oral drugs, this treatment is not widely adopted by investigators. One additional question that had not 
been answered by the trials done so far was whether the antiangiogenic treatment had an impact on mechanisms of acquired resistance, specifically the T790M. At ASCO 2019, data for Erlotinib and Ramucirumab, a VEGF antibody, vs. Erlotinib alone in NSCLC with common mutations were presented. PFS (HR 0.551) and PFS2 (HR 0.69) were highly significantly different, however with immature follow-up, no OS benefit was shown. At progression (acquired resistance), resistance mechanisms were systematically tested, the incidence of $\mathrm{T} 790 \mathrm{M}$ was $43 \%$ in the Erlotinib/Ramucirumab arm, and $47 \%$ in the Erlotinib arm. For a general recommendation of the combination therapy, the final analysis of the study including OS data should be awaited [17].

\section{References}

1. David JK, Valerie R, Jamie C, Bruce J, Nicholas A, et al. (2019) Neoadjuvant Atezolizumab in Resectable Non-Small Cell Lung Cancer (NSCLC): Interim Analysis and Biomarker Data from a Multicenter Study (LCMC3) Journal of Clinical Oncology 37(15): 8503.

2. Cascone T, William WN, Weissferdt A, Lin H, Leung C, et al. (2019) Neoadjuvant nivolumab (N) or nivolumab plus ipilimumab (NI) for resectable non-small cell lung cancer (NSCLC): Clinical and correlative results from the NEOSTAR study. Journal of Clinical Oncology 37(15): 5804.

3. Provencio M, Nadal E, Insa-Molla A, García-Campelo R, Casal J, et al. (2019) Neo-Adjuvant Chemo-Immunotherapy For The Treatment Of Stage Iiia Resectable Non-Small-Cell Lung Cancer (NSCLC): A Phase Ii Multicenter Exploratory Study. NADIM: Neo-Adjuvant Immunotherapy. PASCO 8509.

4. Jhanelle EG, Augusto V, Davey D, David V, Shuji M,et al. (2019) Threeyear Overall Survival Update from the PACIFIC Trial. PASCO.

5. Mark AS, Robert J, Federico C, Tony M, Howard W, et al. (2019] IMpower150: Analysis of Efficacy in Patients With Liver Metastases. Journal of Clinical Oncology 37(15): 9012.

6. Shirish G, Marina CG, Emilio E, Giovanna S, Enriqueta F, et al. (2019) KEYNOTE-189: Updated Overall Survival and Progression After the Next Line of Therapy with Pembrolizumab plus Chemotherapy with Pemetrexed and Platinum vs Placebo plus Chemotherapy for Metastatic nonsquamous NSCLC. Journal of Clinical Oncology 37(15): 9013.

7. Ferdinandos S, Kathryn CA, Matthew DH, Pradnya DP, Melina EM, et al. (2019) Association of STK11/LKB1 genomic alterations with lack of benefit from the addition of pembrolizumab to platinum doublet chemotherapy in non-squamous non-small cell lung cancer Journal of Clinical Oncology 37(15): 102.
8. Marwan Fakih, O'Neil B, Timothy JP, Gerald SF, Jayesh D, et al. (2019) Phase 1 study evaluating the safety, tolerability, pharmacokinetics (PK), and efficacy of AMG 510, a novel small molecule KRASG12C inhibitor, in advanced solid tumors. Journal of Clinical Oncology 37(15): 3003.

9. Paul KP, Remi V, Alexis BC, Enriqueta F, Hiroshi S, et al. (2019) Phase Il study of tepotinib in NSCLC patients with METex14 mutations. Journal of Clinical Oncology 37(15): 9005.

10. Juergen W, Takashi S, Ji-Youn H, Noemi R, Edward BG, et al. (2019) Capmatinib (INC280) in MET $\Delta$ ex14-mutated advanced non-small cell lung cancer (NSCLC): Efficacy data from the phase II GEOMETRY mono-1 study. Journal of Clinical Oncology 37(15): 9004.

11. Robin G, Michael O, Brannon AR, Andrew C, Lukas D, et al. (2019) MET inhibitor resistance in patients with MET exon 14-altered lung cancers. Journal of Clinical Oncology 37(15): 9006.

12. Justin FG, Dae Ho L, Giuseppe C, Robert Charles D, Dong-Wan K, et al. Clinical activity and tolerability of BLU-667, a highly potent and selective RET inhibitor, in patients (pts) with advanced RET-fusion+ non-small cell lung cancer (NSCLC). Journal of Clinical Oncology 37(15): 9008.

13. Pasi AJ, Joel WN, Camidge DR, Alexander IS, Zofia P, et al. (2019) Antitumor activity of TAK-788 in NSCLC with EGFR exon 20 insertions. Journal of Clinical Oncology 37(15): 9007.

14. Vanita N, Amit J, Vijay MP, Anuradha C, Abhishek M, et al. (2019) Phase III randomized trial comparing gefitinib to gefitinib with pemetrexedcarboplatin chemotherapy in patients with advanced untreated EGFR mutant non-small cell lung cancer (gef vs gef $+\mathrm{C}$ ). Journal of Clinical Oncology 37(15): 9001.

15. Noronha V, Patil VM, Joshi A, Menon N, Chougule A, etal. (2019) Gefitinib Versus Gefitinib Plus Pemetrexed and Carboplatin Chemotherapy in EGFR-Mutated Lung Cancer. J Clin Oncol: JC01901154.

16. Roeper J, Falk M, Lueers A, Wedeken K, Stropiep U, et al. Griesinger F TP53 co-mutations in EGFR mutated patients in NSCLC stage IV: A strong predictive factor of ORR, PFS and OS in EGFR mt+ NSCLC: Oncotarget under review.

17. Kazuhiko N, Edward BG, Takashi S, Makoto N, Santiago PA, et al. (2019) RELAY: A multinational, double-blind, randomized Phase 3 study of erlotinib (ERL) in combination with ramucirumab (RAM) or placebo (PL) in previously untreated patients with epidermal growth factor receptor mutation-positive (EGFRm) metastatic non-small cell lung cancer (NSCLC). Journal of Clinical Oncology 37(15): 9000. 
CC (1) This work is licensed under Creative

DOI: 10.19080IJOPRS.2019.04.555634
Your next submission with Juniper Publishers will reach you the below assets

- Quality Editorial service

- Swift Peer Review

- Reprints availability

- E-prints Service

- Manuscript Podcast for convenient understanding

- Global attainment for your research

- Manuscript accessibility in different formats ( Pdf, E-pub, Full Text, Audio)

- Unceasing customer service

Track the below URL for one-step submission https://juniperpublishers.com/online-submission.php 